\title{
Removal of disulfide from acid stress chaperone HdeA does not wholly eliminate structure or function at low pH
}

M. Imex Aguirre-Cardenas ${ }^{1,2}$, Dane H. Geddes-Buehre ${ }^{1}$ and Karin A. Crowhurst ${ }^{1^{*}}$

${ }^{1}$ Department of Chemistry and Biochemistry, California State University Northridge, 18111 Nordhoff St., Northridge CA 91330-8262

${ }^{2}$ Present address: Department of Chemistry, University of California Riverside, 900 University Ave., Riverside, CA 92521

${ }^{*}$ Corresponding author. Email: karin.crowhurst@csun.edu.

CRediT author statement:

Imex Aguirre-Cardenas: Investigation, Visualization, Formal analysis, Writing - Review and Editing. Dane Geddes-Buehre: Investigation, Formal analysis, Writing - Review and Editing. Karin Crowhurst: Conceptualization, Funding acquisition, Supervision, Project administration, Investigation, Formal analysis, Visualization, Writing - Original Draft, Writing - Review and Editing. 


\section{$\underline{\text { Abstract }}$}

HdeA is an acid-stress chaperone that operates in the periplasm of various strains of pathogenic gramnegative bacteria. Its primary function is to prevent irreversible aggregation of other periplasmic proteins when the bacteria enter the acidic environment of the stomach after contaminated food is ingested; its role is therefore to help the bacteria survive long enough to enter and infect the intestines. The mechanism of operation of HdeA is unusual in that this helical homodimer is inactive when folded at neutral $\mathrm{pH}$ but becomes activated at low $\mathrm{pH}$ after the dimer dissociates and becomes partially unfolded. Studies with chemical reducing agents have previously suggested that the intramolecular disulfide bond of HdeA aids in the maintenance of residual structure at low $\mathrm{pH}$; it is believed that this residual structure is important for clustering exposed hydrophobic residues together for the purpose of binding unfolded client proteins. In order to explore its role in HdeA structure and chaperone function we performed a conservative cysteine to serine mutation of the disulfide. We found that, although residual structure is greatly diminished at $\mathrm{pH} 2$ without the disulfide, it is not completely lost; conversely, the mutant is almost completely random coil at $\mathrm{pH} 6$. Aggregation assays showed that mutated HdeA, although less successful as a chaperone than wild type, still maintains a surprising level of function. These studies highlight that we still have much to learn about the factors that stabilize residual structure at low $\mathrm{pH}$ and the role of disulfide bonds.

Keywords: acid-stress protein; chaperone protein; protein unfolding; disulfide bond; NMR chemical shifts; aggregation assay 


\section{Introduction}

HdeA is an ATP-independent chaperone protein [2] found in the periplasm of several pathogenic bacteria including Shigella flexneri, Escherichia coli and Brucella abortus [3-5]. It, along with sister protein $\mathrm{HdeB}$, helps to prevent the irreversible aggregation of other periplasmic proteins when the organism encounters a low pH environment (found in the stomach). In this way, HdeA aids in the survival of these bacteria so that they can enter the intestines of the host and cause dysentery, a disease which affects at least 120 million people each year [6, 7]. Folded HdeA is a helical homodimer (Figure 1). One of its particularly unusual characteristics is that its folded state (at near-neutral $\mathrm{pH}$ ) is its inactive state; once the bacteria enter a low pH environment (below approximately $\mathrm{pH} 3$ ) HdeA unfolds and assumes its active role as a chaperone $[3,8]$.

HdeA contains one intramolecular disulfide bond between residues 18 and 66. It has been conjectured that it may help to bring two hydrophobic sites into close contact at low $\mathrm{pH}$, thereby facilitating chaperone activity by enabling the formation of an extended client binding site [9]. Although its importance is mentioned in several publications on $\operatorname{HdeA}[8,10-12]$, relatively little investigation has been done on the C18-C66 disulfide bond. Hong et al. [8] originally reported that the chaperone activity

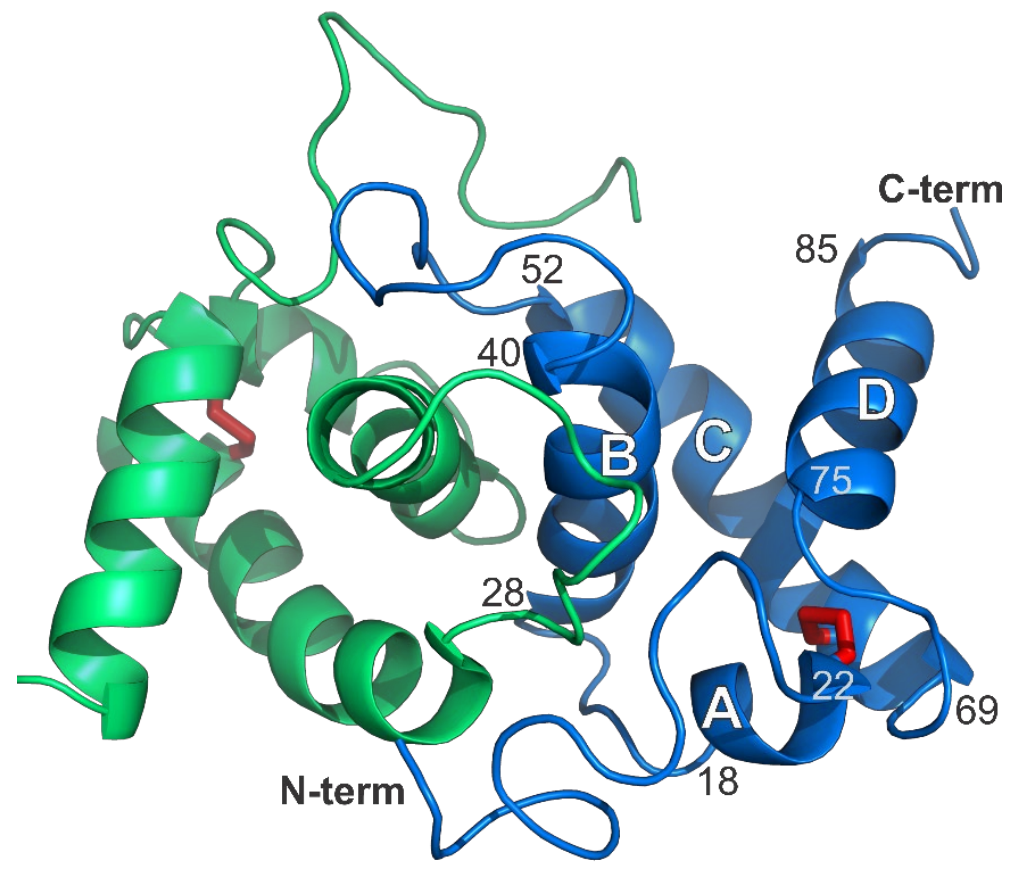

Figure 1. Labeled structure of the folded HdeA homodimer (PDB ID 5WYO) [2]. The blue monomer shows the locations of helices $A-D$ (including residue number ranges) and the $\mathrm{N}$ - and $\mathrm{C}$-termini. The disulfide bond between residues 18 and 66 is shown in red. 
of HdeA was unaffected by a reduction of the disulfide bond at low $\mathrm{pH}$, but because they utilized dithiothreitol (DTT), which is functional only in the $\mathrm{pH}$ range $6.5-9.0$, these observations were not valid. Tapley et al. [9] apparently demonstrated via light-scattering studies at $320 \mathrm{~nm}$ that, at low $\mathrm{pH}$, reduced HdeA cannot act as a chaperone, although there are questions about those studies, given that DTT was once again used as the reducing agent. Finally, Zhai et al. [13] used TCEP (tris(2carboxyethyl)phosphine), which is active over a wide $\mathrm{pH}$ range $(1.5-8.5)$, to evaluate the chaperone activity of HdeA with a reduced disulfide at low $\mathrm{pH}$, using SDS-PAGE to detect client protein aggregates. The authors suggest that the chaperone activity of reduced HdeA is heavily compromised, although their gel seems to show almost equal quantities of soluble and precipitated client protein in the presence of TCEP-treated HdeA [13]. In addition, it is unclear whether HdeA remains reduced during the assay, since the authors describe a ten-fold dilution of the TCEP at one stage.

Considering that researchers assert the presence of the disulfide is crucial for HdeA chaperone activity but have not demonstrated this definitively, we decided to prepare a double mutant of HdeA in which the cysteines were conservatively mutated to serines. These mutations eliminate the possibility of disulfide bond formation without eliminating side chain hydrogen bond capabilities and allow for studies in the absence of chemical reductants. Our results show that the loss of the disulfide bond eliminates the ability of HdeA-C18S-C66S (also called C18S-C66S) to fold at neutral pH. The mutant has nearrandom coil structure at $\mathrm{pH} 6.0$, but surprisingly gains some secondary structure content at low $\mathrm{pH}$, although it is still lower than that of wild type HdeA at pH 2.0. Additionally, a comparison of chaperone activity of C18S-C66S versus TCEP-reduced wild type on client protein malate dehydrogenase (MDH) indicates that both have notable (although not complete) success in keeping the client protein soluble.

\section{Materials and Methods}

Isotopes were obtained from Cambridge Isotope Laboratories, and chromatography columns 
were from GE Lifesciences.

\section{Preparation of HdeA}

Site-directed mutagenesis was executed using the QuikChange Lightning kit from Agilent. HdeA-C18S-C66S was expressed and purified as outlined previously for wild type $\operatorname{HdeA}[11,14,15]$, with the exception that a Superdex 75 HiLoad 26/600 column was required rather than the usual HR 10/30 column. All samples were uniformly ${ }^{13} \mathrm{C} /{ }^{15} \mathrm{~N}$ labeled.

\section{NMR experiments}

C18S-C66S samples were prepared for NMR through dialysis into $50 \mathrm{mM}$ citrate buffer at the desired $\mathrm{pH}$ and had final concentrations in the range of $0.1-1.0 \mathrm{mM}$. Since no chemical shift changes were observed as a function of sample concentration, we were not concerned about the impact of varied protein concentration on the results presented. Reduced samples of wild type HdeA contained $5 \mathrm{mM}$ tris(2-carboxyethyl)phosphine (TCEP); in order to obtain spectra at pH 6.0 the protein sample was first reduced in the unfolded state at low $\mathrm{pH}$ and then dialyzed to 6.0 in the presence of TCEP. NMR data were obtained at $25^{\circ} \mathrm{C}$ on an Agilent DD2 $600 \mathrm{MHz}$ spectrometer equipped with a triple resonance probe. All raw data were processed using NMRPipe/NMRDraw $[16,17]$ and the resulting spectra were viewed and analyzed using NMRViewJ [18, 19].

Chemical shift assignment. Because C18S-C66S is unfolded at pH 6.0 and 2.0, backbone chemical shift assignments at both pHs required $\mathrm{HNCaCb}, \mathrm{CbCa}(\mathrm{CO}) \mathrm{NH}$ and $\mathrm{HNN}[20]$ experiments; additionally, $\mathrm{HNCO}$ and $\mathrm{HN}(\mathrm{Ca}) \mathrm{CO}$ experiments were required at $\mathrm{pH}$ 6.0. The assignment data have been deposited at the BioMagResBank (BMRB), acquisition number 50437. Wild type HdeA assignments at pH 2.0 (BMRB acquisition number 50421, [1]) and pH 6.0 (BMRB acquisition number 19165, [14]) were reported previously, and assignments of spectra from wild type HdeA in TCEP were made by overlaying 
with mutant spectra.

Backbone amide chemical shift differences (CSDs, or $\Delta \delta$ ) were calculated using the equation:

$$
\Delta \delta=\sqrt{\frac{(\Delta H)^{2}+\left(\frac{\Delta N}{7}\right)^{2}}{2}}
$$

where $\Delta \mathrm{H}$ and $\Delta \mathrm{N}$ refer to the differences in backbone ${ }^{1} \mathrm{H}_{\mathrm{N}}$ and ${ }^{15} \mathrm{~N}$ chemical shifts for a given residue between different $\mathrm{pHs}$ or between wild type and mutant at a specific $\mathrm{pH}$.

Secondary structure propensity. SSP analysis was performed on HdeA and HdeA-C18S-C66S using only $\mathrm{C} \alpha$ and $\mathrm{C} \beta$ chemical shifts, as recommended for an unfolded protein [21].

\section{Aggregation assays}

The chaperone activity of C18S-C66S to prevent or rescue aggregation of malate dehydrogenase $(\mathrm{MDH})$ was tested at $\mathrm{pH} 2.0$ and 6.0, using Aggregation Buffer consisting of $20 \mathrm{mM}$ citrate, $100 \mathrm{mM}$ sodium chloride and $150 \mathrm{mM}$ ammonium sulfate [13]. All samples contained $10 \mu \mathrm{M} \mathrm{MDH}$ and some contained $30 \mu \mathrm{M}$ wild type HdeA or mutant, plus $5 \mathrm{mM}$ TCEP when appropriate. At $\mathrm{pH} 2.0$, the mixtures were incubated at $37^{\circ} \mathrm{C}$ for one hour, then centrifuged at $14,000 \mathrm{xg}$ for 10 minutes to separate the supernatant and pellet. The pellets were washed once with Aggregation Buffer and centrifuged again (to remove surface supernatant); the supernatants were partially neutralized with 0.13 volumes of $0.5 \mathrm{M}$ sodium phosphate, $\mathrm{pH} 8$ in advance of analysis via SDS-PAGE. At pH 6.0, MDH was pre-treated with $2 \mathrm{M}$ guanidinium hydrochloride in Aggregation Buffer and incubated at $100^{\circ} \mathrm{C}$ for 20 minutes to ensure $\mathrm{MDH}$ was denatured before mixing with HdeA (wild type or mutant), and to test the ability of the chaperone to "rescue" aggregated $\mathrm{MDH}$ at $\mathrm{pH}$ 6.0. The mixtures were incubated at $37^{\circ} \mathrm{C}$ for one hour, then centrifuged to separate the supernatant and pellet. Pellets were washed once with Aggregation Buffer and centrifuged again. All samples were run on 15\% SDS-PAGE gels. 


\section{$\underline{\text { Results and Discussion }}$}

\section{Chemical shift assignment}

Upon overlaying the ${ }^{15} \mathrm{~N}-\mathrm{HSQC}$ of C18S-C66S with wild type HdeA at $\mathrm{pH} 2.0$, it was clear that the mutations significantly alter the residual low-pH structure of HdeA (Figure S1a). After comparing the spectra of C18S-C66S at pH 2.0 and 6.0 (Figure S1b) it was also clear that the loss of the disulfide bond results in unfolded protein at both pHs, but the ensembles of structures are not the same. To aid in chemical shift assignment of unfolded protein at each $\mathrm{pH}$, we employed the 3D HNN experiment [20] in addition to the $\mathrm{HNCaCb} / \mathrm{CbCa}(\mathrm{CO}) \mathrm{NH}$ and, at $\mathrm{pH} 6.0$, the $\mathrm{HN}(\mathrm{Ca}) \mathrm{CO} / \mathrm{HNCO}$ suites of experiments. In the end we achieved near-complete backbone assignment: $99 \%$ of the backbone ${ }^{1} \mathrm{H} /{ }^{15} \mathrm{~N}$ atoms and $99 \%$ of the $\mathrm{C} \alpha / \mathrm{C} \beta$ atoms at $\mathrm{pH} 2.0$, as well as $95 \%$ of the backbone ${ }^{1} \mathrm{H} /{ }^{15} \mathrm{~N}$ atoms, $99 \%$ of the $\mathrm{C} \alpha / \mathrm{C} \beta$ atoms and $100 \%$ of the $\mathrm{C}(\mathrm{O})$ atoms at $\mathrm{pH} 6.0$ were successfully assigned. Missing assignments at $\mathrm{pH} 2.0$ were located at P12 $(\mathrm{C} \beta), \mathrm{G} 34(\mathrm{C} \alpha)$ and $\mathrm{K} 87\left({ }^{1} \mathrm{H} /{ }^{15} \mathrm{~N}\right)$, while at $\mathrm{pH} 6.0$ missing assignments were at $\mathrm{D} 2\left({ }^{1} \mathrm{H} /{ }^{15} \mathrm{~N}\right)$, K42 $\left({ }^{1} \mathrm{H} /{ }^{15} \mathrm{~N}\right), \mathrm{K} 44(\mathrm{C} \beta), \mathrm{D} 83\left({ }^{1} \mathrm{H} /{ }^{15} \mathrm{~N}\right)$ and $\mathrm{I} 85\left({ }^{1} \mathrm{H} /{ }^{15} \mathrm{~N}\right)$. The data have been deposited the BioMagResBank (BMRB), acquisition number 50437.

\section{Chemical shift differences indicate some long-range impacts of disulfide removal.}

The sites of the largest differences $(\Delta \delta$, or CSD) in backbone amide chemical shift were evaluated. When comparing wild type HdeA to C18S-C66S at $\mathrm{pH} 2.0$, it is noteworthy that the amide shifts of the first and last 15 residues in the protein are essentially indistinguishable (Figure 2a). As one might expect, the largest chemical shift differences can be found near (but not always at) the mutated cysteines (Figure $\mathbf{2 b}$ ). In a trend that is consistent with other experiments we have done [1, 11], the largest changes are on the C-terminal side of position 18 (residues $20-25$ ) and the $\mathrm{N}$-terminal side of 
position 66 (residues 57-65). Within the folded structure, portions of these two regions (residues $20-$ 25 and $62-65$ ) are adjacent to each other; this proximity seems to be maintained in the unfolded state
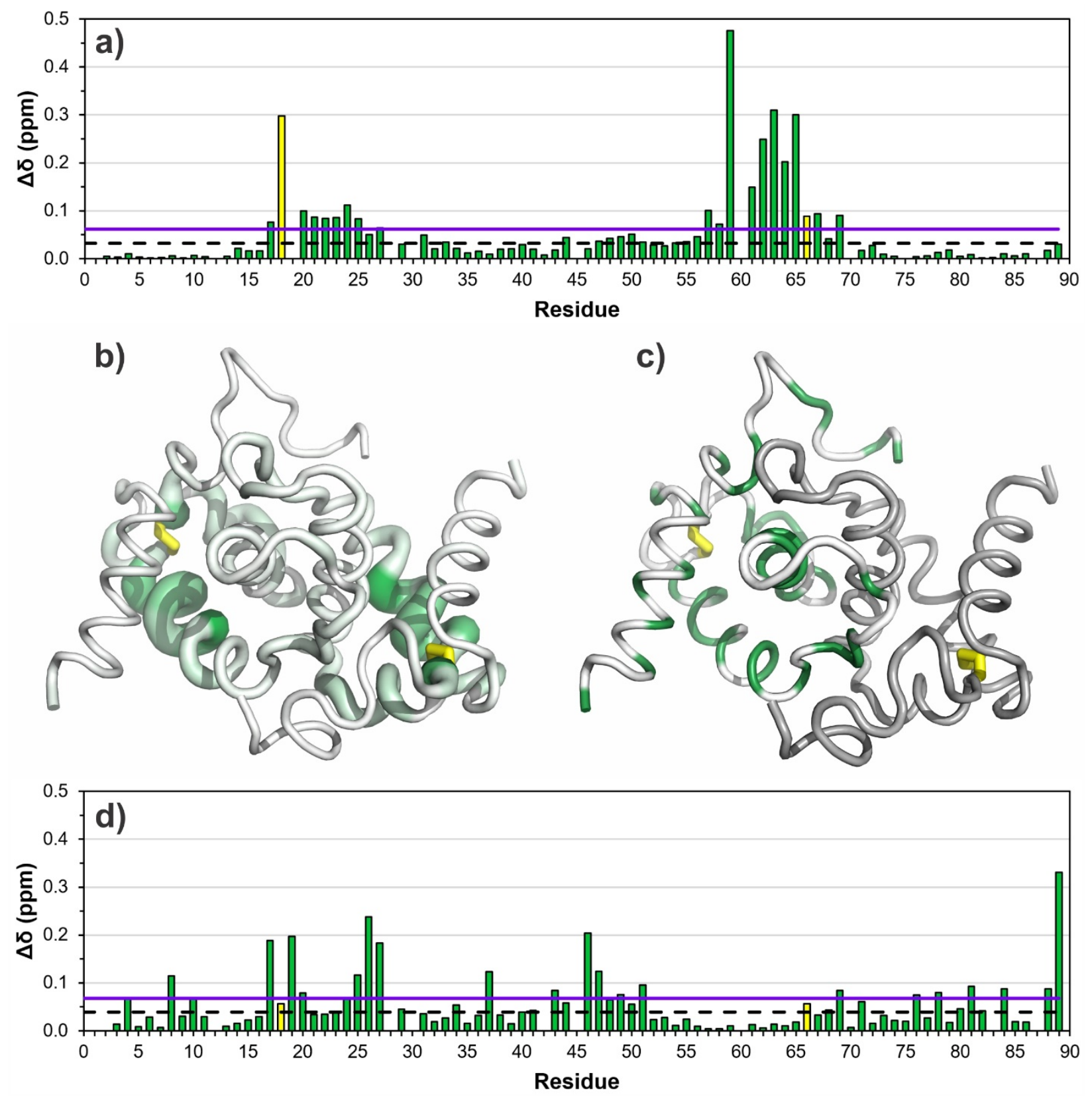

Figure 2. Evaluation of the amide ${ }^{1} \mathrm{H}$ and ${ }^{15} \mathrm{~N}$ chemical shift differences (CSD, or $\Delta \delta$ ), as a function of residue. a) CSD between wild type $\mathrm{HdeA}$ and $\mathrm{C} 18 \mathrm{~S}-\mathrm{C} 66 \mathrm{~S}$ at $\mathrm{pH}$ 2.0. The positions of the mutation sites are colored in yellow. The black dashed horizontal line corresponds to the average $\Delta \delta$ (minus $10 \%$ outliers), and the purple horizontal line indicates one standard deviation above the mean. b) The $\Delta \delta$ values from a) are plotted on the folded structure of HdeA. Residues with higher $\Delta \delta$ are shown with darker green and larger cartoon radius, and the site of the wild type disulfide is colored yellow. c) Positions of hydrophobic groups in HdeA, colored green on one chain. d) CSD between C18S-C66S at pH 6.0 and 2.0. 
of the wild type, primarily due to the disulfide tether [11]. However, residues 57-61 extend along the entire C-terminal half of helix C, far from residues $20-25$ and far from the site of the removed disulfide in the mutant (Figure $\mathbf{2 b}$ ). When assessing secondary structure propensities (based on chemical shift assignments), the loss of the disulfide not only eliminates the helical propensity of helix $\mathrm{C}$ that is seen in the wild type, its secondary structure flips to weak $\beta$-sheet content (Figure $\mathbf{3}$ and Table S1); this, or an allosteric effect, could explain the large chemical shift changes.

Like the groups described above, residues on the opposite side of each cysteine, at the Nterminal side of position 18 (including $14-16$ ) and the C-terminal side of position 66 (residues $70-74$ ), are also adjacent to each other in the folded protein; however, these segments of the mutant do not have significant differences in chemical shift at pH 2.0 compared to wild type. In folded wild type HdeA, this region acts like a clasp that is opened at low $\mathrm{pH}$ to expose hydrophobic client binding sites as part of its chaperone activation [1]. Refer to Figure $\mathbf{2 c}$ for the positions of each hydrophobic residue in HdeA. The chemical shift similarities imply structural similarities in this region between wild type and C18S$\mathrm{C66S}$ at $\mathrm{pH} 2.0$; these results support the notion that this region of the protein is indeed open and less structured in the wild type when the clasp is released at low $\mathrm{pH}$.

Upon initial inspection, the chemical shift differences between C18S-C66S at pH 6.0 and 2.0

(Figure $\mathbf{2 d}$ ), seem to have no pattern to the magnitude of $\Delta \delta$ value. However, upon closer inspection, it becomes clear that $14 / 19$ residues with $\Delta \delta>1$ standard deviation above the mean are aspartate or glutamate, all of which undergo neutralization in transitioning from $\mathrm{pH} 6.0$ to 2.0. The remaining residues likely have large chemical shift perturbations due to their proximity to these ionizable groups.

\section{Secondary structure propensity analysis shows that the mutant maintains some structure at low pH but is virtually random coil in near-neutral conditions.}

As mentioned briefly above, the chemical shift data were also used to calculate and compare 
secondary structure propensities (SSP). Using the recommendation by Marsh et al. [21], only C $\alpha$ and C $\beta$ shifts were used to calculate SSP values for the unfolded proteins. Figure $\mathbf{3}$ shows an overlay of SSP values as a function of residue number for wild type and C18S-C66S at $\mathrm{pH} 2.0$, as well as C18S-C66S at pH 6.0. See Table S1 for the numerical values and Figure S2 for a plot showing the change in mutant SSP ( $\triangle \mathrm{SSP}$ ) between $\mathrm{pH} 2.0$ and 6.0 as a function of residue number. As has been observed in previous publications $[1,12]$, even at $\mathrm{pH} 2.0$ the "unfolded" wild type maintains notable residual helical secondary structure, albeit in slightly shifted positions compared to the folded structure [1]. However, what is truly remarkable when evaluating the SSP values for C18S-C66S is that the mutant has more structural content at low pH compared to near-neutral conditions (Figure 3). At pH 6.0 C18S-C66S has extremely low SSP values, almost exclusively in the -0.1 to 0.1 range, suggesting that the protein is very close to random coil. However, at pH 2.0, the SSP values in the mutant are significantly strengthened in almost every region of the protein compared to $\mathrm{pH} 6.0$; its helical secondary structure propensity even

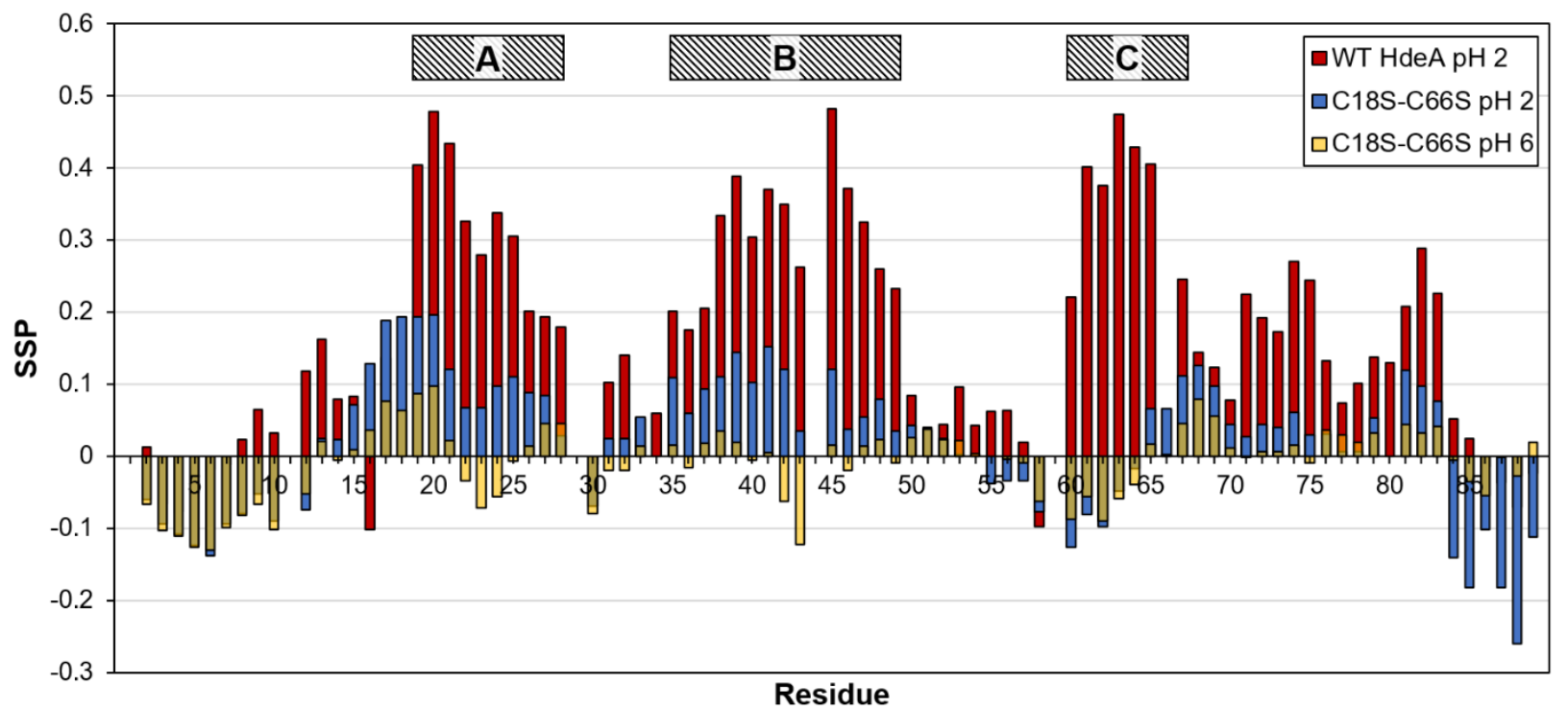

Figure 3. Plot of secondary structure propensities (SSP) as a function of residue number for wild type HdeA at pH 2.0 (red) and HdeA-C18S-C66S at pH 2.0 (blue) and pH 6.0 (yellow, shown as 50\% transparent in order to show values from the other samples underneath). All data were calculated using only $\mathrm{C} \alpha$ and $\mathrm{C} \beta$ chemical shifts, as recommended for unfolded proteins. Positive SSP values indicate helical secondary structure, with maximum possible value of 1.0 (indicating $100 \%$ helical propensity) and negative SSP values indicate $\beta$-sheet secondary structure (where a value of -1 would indicate $100 \% \beta$-sheet forming propensity). Approximate positions of residual helix structure in wild type $\mathrm{HdeA}$ at low $\mathrm{pH}$ are indicated at the top of the plot [1]. 
outperforms wild type at several residues on the $\mathrm{N}$-terminal side of the C18S mutation. Additionally, at $\mathrm{pH} 2.0$ the C18S-C66S mutant displays notable $\beta$ structure propensity at the C-terminus, while wild type shows no persistent secondary structure in that region (Figure 3 and Table S1). C18S-C66S also displays greater $\beta$ propensity at the $\mathrm{N}$-terminus at both $\mathrm{pHs}$ compared to unfolded wild type, suggesting that the $\mathrm{N}$ - and $\mathrm{C}$-termini of the mutant may have an increased tendency to form semi-stable $\beta$-sheet structures with each other; this is even higher than the tendency we observed when modeling the unfolded wild type with MD simulations [11]. In trying to explain the gain of structure in the mutant at $\mathrm{pH} 2.0$ compared to $\mathrm{pH} 6.0$ we wondered whether there is prior experimental evidence that hydrogen bonds are generally stronger at low $\mathrm{pHs}$ compared to neutral $\mathrm{pH}$. If this were so, we could argue that even weak hydrogen bonds between S18 and S66 (or between other residues) in the mutant could help maintain a portion of the secondary structure that is observed in the wild type at $\mathrm{pH}$ 2.0. Alternatively, there may be other features, in addition to the disulfide in wild type HdeA, that help the protein maintain a partially folded conformation at low $\mathrm{pH}$ and that are strengthened in the mutant. Unfortunately, we have yet to find published evidence of either hypothesis; the phenomenon may be worth pursuing computationally.

\section{C18S-C66S has similar (but not identical) chemical shifts to wild type HdeA in TCEP.}

We were curious to see whether our double mutant behaved similarly to wild type HdeA in the presence of the reducing agent TCEP. When comparing the CSDs between reduced wild-type and C18S-C66S at both pH 2.0 and 6.0 (Figures S3 and S4), the presence of TCEP clearly results in a similar ensemble of unfolded structures as those observed for the double mutant. The chemical shift perturbations are, overall, an order of magnitude smaller than comparisons between unfolded, oxidized wild type HdeA and C18S-C66S at pH 2.0 (compare Figure 2a and S4). Even so, the CSDs at each pH follow distinctly different trends. At pH 6.0, the CSD values between wild type in TCEP and C18S-C66S 
are very low, except in the region immediately surrounding the mutation sites (as one might expect). However, at $\mathrm{pH} 2.0$, the average CSD is more than double what is observed at $\mathrm{pH} 6.0$, and although the largest values are also near the mutation sites, there are other notably high values near the $\mathrm{N}$-terminus and between residues $47-51$ (Figure S4a). Neither of these regions are close to the disulfide in the folded state, but the $\mathrm{N}$-terminus of one protomer is in contact with residues $47-51$ in the other protomer of the folded dimer. In looking at the residues with the highest CSD in those two regions, five out of the six are aspartates. This suggests that TCEP may specifically interact with aspartates in solution, altering their backbone amide chemical shifts, but we cannot explain why the other aspartates (or glutamates) in the protein are not similarly affected.

\section{Loss of the disulfide does not wholly eliminate chaperone activity in HdeA.}

We were interested to evaluate the chaperone activity of C18S-C66S, and to determine whether the activity of wild type HdeA in TCEP was comparable. It has been suggested previously that the disulfide may have a crucial role in chaperone activation by clustering hydrophobic patches in HdeA and creating a larger client binding site in the partially unfolded state [9]. It was therefore expected that neither the mutant nor chemically reduced wild type HdeA would have the ability to protect a client protein from aggregation. We performed aggregation assays at $\mathrm{pH} 2.0$ and 6.0, employing methods similar to those reported by other groups, using malate dehydrogenase (MDH) as a stand-in for a client protein $[9,13,22,23]$. Figures $\mathbf{S} 5$ and $\mathbf{S} 6$ show the gel images and plots of quantified band density, and Table 1 summarizes the density values. The results at $\mathrm{pH} 6.0$ are as one might expect - even wild type HdeA is not expected to rescue aggregated MDH since it is folded and generally inactive as a chaperone at that $\mathrm{pH}$ (Figure S5). At pH 2.0, $\mathrm{MDH}$ without chaperone is found almost exclusively in the pellet and $\mathrm{MDH}$ in the presence of wild type HdeA is found almost exclusively in the supernatant, as expected

(Figure S6). The results become interesting when evaluating the chaperone activity for C18S-C66S and 
Table 1. Relative volume of the gel bands from the aggregation assays performed at $\mathrm{pH} 6.0$ and 2.0 .

\begin{tabular}{|c|c|c|}
\hline & pH 6.0 & pH 2.0 \\
\hline Pa - MDH only & $96.2^{b}$ & 89.4 \\
\hline $\mathrm{S}^{\mathrm{a}}-\mathrm{MDH}$ only & 3.8 & 10.6 \\
\hline$P-W T^{c} H d e A$ & 94.6 & 1.5 \\
\hline S - WT HdeA & 5.4 & 98.5 \\
\hline P - WT HdeA + TCEP & 91.4 & 59.8 \\
\hline S - WT HdeA + TCEP & 8.6 & 40.2 \\
\hline $\mathrm{P}-\mathrm{HdeA}-\mathrm{C} 18 \mathrm{~S}-\mathrm{C} 66 \mathrm{~S}$ & 95.5 & 63.5 \\
\hline S-HdeA-C18S-C66S & 4.5 & 36.5 \\
\hline $\mathrm{P}-\mathrm{HdeA}-\mathrm{C} 18 \mathrm{~S}-\mathrm{C} 66 \mathrm{~S}+\mathrm{TCEP}$ & 95.5 & 64.9 \\
\hline S - HdeA-C18S-C66S + TCEP & 4.5 & 35.1 \\
\hline \multicolumn{3}{|c|}{$\begin{array}{l}\text { a } \mathrm{P} \text { and } \mathrm{S} \text { labels represent lanes containing pellet (aggregated } \mathrm{MDH} \text { ) or } \\
\text { supernatant (soluble } \mathrm{MDH} \text { ), respectively. } \\
{ }^{\mathrm{b}} \text { Numbers estimated using volume integration data from Bio-Rad Image Lab } \\
\text { software. Values correspond to \% relative volume (each pellet-supernatant pair } \\
\text { adds up to } 100 \% \text { ). }\end{array}$} \\
\hline
\end{tabular}

HdeA plus TCEP; although there is a sizeable portion of insoluble client protein, at least one-third of the MDH can be found in the supernatant of each sample (Table 1 and Figure S6). This implies that, while the disulfide bond is important to the success of HdeA as a chaperone, the lack of disulfide does not fully eliminate its capabilities.

Although the results show that wild type HdeA plus TCEP has similar chaperone properties as the C18S-C66S mutant, the NMR data indicate there are still differences in the conformational ensembles (Figures S3a and S4a). Given these results, we argue it is advantageous to use the mutant in any future studies, since it eliminates the variability and complication of employing a chemical additive which may also have unintended effects on other solution components, such as a client protein.

\section{Results provide evidence for the maintenance of some hydrophobic clustering in mutant HdeA.}

Considering that $\mathrm{HdeA}$ maintains some chaperone activity at low $\mathrm{pH}$, even in the absence of the disulfide, it is worthwhile to take a deeper look at the data. We have already suggested that there may 
be hydrogen bonding between the serines in C18S-C66S that can help maintain some residual structure (and therefore chaperone activity) at $\mathrm{pH} 2.0$, but this alone is unlikely to fully explain our assay results. Helix C in wild type HdeA contains numerous hydrophobic groups and constitutes a portion of client binding site I proposed by Yu et al. [12]. However, at pH 2.0 a large segment of this region shows significant chemical shift perturbation in C18S-C66S compared to wild type (Figure 2a), and most of its secondary structure propensity is lost (Figure 3). These data therefore suggest that there is little residual hydrophobic clustering in this region and therefore does not contribute to the protein's chaperone activity. On the other hand, when evaluating the region corresponding to helix B and the BC loop (as seen in folded $\mathrm{HdeA}$ ), the mutant has very similar amide chemical shifts to that of wild type at $\mathrm{pH} 2.0$ (Figure 2), and it maintains some residual helical structure (Figure 3). This region is the location of the proposed client binding site II [12], and is also the same segment that is believed to become accessible (due to the opening of the clasp region) when the wild type unfolds and becomes chaperone-active [1]. In addition, the SSP data show that there is increased $\beta$ structure propensity at both the $\mathrm{N}$ - and Ctermini in C18S-C66S (Figure 3) compared to wild type; previous studies have provided evidence that the termini transiently form a $\beta$-sheet structure as part of HdeA's chaperone activation [11]. Taken together, it is possible that this strengthened $\beta$-sheet formation (compared to wild type) helps C18S-C66S maintain a more compact shape, subsequently preserving the hydrophobic cluster in the client binding site near residues $28-39$ and possibly explaining the partial chaperone activity of the mutant at pH 2.0. Future studies will investigate the importance to chaperone activity of this $\beta$-sheet formation as well as the specific location of the disulfide.

\section{Conclusions}

Replacement of the cysteines that form the disulfide bond in HdeA with serines results in a nearrandom coil structure at $\mathrm{pH}$ 6.0, but notably higher structural content at $\mathrm{pH} 2.0$. HdeA-C18S-C66S also 
retains greater low-pH chaperone activity than previously suggested. Both low-pH results for the mutant are unexpected, highlighting the need for more investigation of positions 18 and 66, located at a "pinch point" of the HdeA structure [1], as well as other residues in the vicinity and at the $\mathrm{N}$ - and C-termini that may collectively maintain partial chaperone activity when the cysteines are removed or reduced. These results underscore the complexities of the structure-function relationship in this acid stress chaperone and may open new areas of inquiry into the role of long-range disulfide bonds in small proteins.

\section{Acknowledgements}

We gratefully acknowledge the NIH for research support (SC3-GM116745) and the NSF for funding the purchase of our NMR spectrometer (CHE-1040134). Sincere thanks also go to Ranjith Muhandiram (University of Toronto) for assistance with the HNN assignment experiment and for helpful discussions.

\section{$\underline{\text { References }}$}

[1] Widjaja, M.A., Gomez, J.S., Benson, J.M. \& Crowhurst, K.A. (2021). Detection of key sites of dimer dissociation and unfolding initiation during activation of acid-stress chaperone HdeA at low pH. Biochim Biophys Acta Proteins Proteom. 1869, 140576. DOI: 10.1016/j.bbapap.2020.140576.

[2] Yu, X.C., Yang, C., Ding, J., Niu, X., Hu, Y. \& Jin, C. (2017). Characterizations of the Interactions between Escherichia coli Periplasmic Chaperone HdeA and Its Native Substrates during Acid Stress. Biochemistry. 56, 5748-5757. DOI: 10.1021/acs.biochem.7b00724.

[3] Gajiwala, K.S. \& Burley, S.K. (2000). HDEA, a periplasmic protein that supports acid resistance in pathogenic enteric bacteria. J Mol Biol. 295, 605-612. DOI: 10.1006/jmbi.1999.3347. 
[4] Valderas, M.W., Alcantara, R.B., Baumgartner, J.E., Bellaire, B.H., Robertson, G.T., Ng, W.L., Richardson, J.M., Winkler, M.E., et al. (2005). Role of HdeA in acid resistance and virulence in Brucella abortus 2308. Vet Microbiol. 107, 307-312. DOI: 10.1016/j.vetmic.2005.01.018.

[5] Waterman, S.R. \& Small, P.L. (1996). Identification of sigma S-dependent genes associated with the stationary-phase acid-resistance phenotype of Shigella flexneri. Mol Microbiol. 21, 925-940. DOI: 0.1046/j.1365-2958.1996.00058.x.

[6] (WHO), W.H.O. Global Health Estimates 2016: Deaths by Cause, Age, Sex, by Country and by Region, 2000-2016. Geneva: World Health Organization (WHO); 2018.

[7] Khalil, I.A., Troeger, C., Blacker, B.F., Rao, P.C., Brown, A., Atherly, D.E., Brewer, T.G., Engmann, C.M., et al. (2018). Morbidity and mortality due to shigella and enterotoxigenic Escherichia coli diarrhoea: the Global Burden of Disease Study 1990-2016. Lancet Infect Dis. 18, 1229-1240. DOI: 10.1016/S1473-3099(18)30475-4.

[8] Hong, W., Jiao, W., Hu, J., Zhang, J., Liu, C., Fu, X., Shen, D., Xia, B., et al. (2005). Periplasmic protein HdeA exhibits chaperone-like activity exclusively within stomach $\mathrm{pH}$ range by transforming into disordered conformation. J Biol Chem. 280, 27029-27034. DOI: 10.1074/jbc.M503934200.

[9] Tapley, T.L., Korner, J.L., Barge, M.T., Hupfeld, J., Schauerte, J.A., Gafni, A., Jakob, U. \& Bardwell, J.C. (2009). Structural plasticity of an acid-activated chaperone allows promiscuous substrate binding. Proc Natl Acad Sci U S A. 106, 5557-5562. DOI: 10.1073/pnas.0811811106.

[10] Ahlstrom, L.S., Law, S.M., Dickson, A. \& Brooks, C.L., 3rd. (2015). Multiscale modeling of a conditionally disordered pH-sensing chaperone. J Mol Biol. 427, 1670-1680. DOI: 10.1016/j.jmb.2015.01.002.

[11] Pacheco, S., Widjaja, M.A., Gomez, J.S., Crowhurst, K.A. \& Abrol, R. (2020). The complex role of the $\mathrm{N}$-terminus and acidic residues of $\mathrm{HdeA}$ as $\mathrm{pH}$-dependent switches in its chaperone function. Biophysical Chemistry. 264, 106406. DOI: 10.1016/j.bpc.2020.106406. 
[12] Yu, X.C., Hu, Y., Ding, J., Li, H. \& Jin, C. (2019). Structural basis and mechanism of the unfoldinginduced activation of HdeA, a bacterial acid response chaperone. J Biol Chem. 294, 3192-3206. DOI: 10.1074/jbc.RA118.006398.

[13] Zhai, Z., Wu, Q., Zheng, W., Liu, M., Pielak, G.J. \& Li, C. (2015). Roles of structural plasticity in chaperone HdeA activity are revealed by ${ }^{19} \mathrm{~F}$ NMR. Chem Sci. 7, 2222-2228. DOI: $10.1039 / \mathrm{c} 5 \mathrm{sc0} 04297 f$.

[14] Crowhurst, K.A. (2014). ${ }^{13} \mathrm{C},{ }^{15} \mathrm{~N}$ and ${ }^{1} \mathrm{H}$ backbone and side chain chemical shift assignment of acidstress bacterial chaperone HdeA at pH 6. Biomol NMR Assign. 8, 319-323. DOI: 10.1007/s12104013-9508-0.

[15] Garrison, M.A. \& Crowhurst, K.A. (2014). NMR-monitored titration of acid-stress bacterial chaperone HdeA reveals that Asp and Glu charge neutralization produces a loosened dimer structure in preparation for protein unfolding and chaperone activation. Protein Sci. 23, 167-178. DOI: 10.1002/pro.2402.

[16] Delaglio, F. NMRPipe / NMRDraw. 9.6 ed. North Potomac, MD: NMR Science; 2018.

[17] Delaglio, F., Grzesiek, S., Vuister, G.W., Zhu, G., Pfeifer, J. \& Bax, A. (1995). NMRPipe: a multidimensional spectral processing system based on UNIX pipes. J Biomol NMR. 6, 277-293. DOI: 10.1007/bf00197809.

[18] Johnson, B.A. NMRViewJ. 9.2 ed. Newark, NJ: One Moon Scientific, Inc.; 2016.

[19] Johnson, B.A. \& Blevins, R.A. (1994). NMR View - A computer-program for the visualization and analysis of NMR data. J Biomol NMR. 4, 603-614. DOI: 10.1007/BF00404272.

[20] Panchal, S.C., Bhavesh, N.S. \& Hosur, R.V. (2001). Improved 3D triple resonance experiments, HNN and $\mathrm{HN}(\mathrm{C}) \mathrm{N}$, for $\mathrm{HN}$ and ${ }^{15} \mathrm{~N}$ sequential correlations in $\left({ }^{13} \mathrm{C},{ }^{15} \mathrm{~N}\right)$ labeled proteins: application to unfolded proteins. J Biomol NMR. 20, 135-147. DOI: 10.1023/A:1011239023422. 
[21] Marsh, J.A., Singh, V.K., Jia, Z. \& Forman-Kay, J.D. (2006). Sensitivity of secondary structure propensities to sequence differences between alpha- and gamma-synuclein: implications for fibrillation. Protein Sci. 15, 2795-2804. DOI: 10.1110/ps.062465306.

[22] Foit, L., George, J.S., Zhang, B.W., Brooks, C.L., 3rd \& Bardwell, J.C. (2013). Chaperone activation by unfolding. Proc Natl Acad Sci U S A. 110, E1254-1262. DOI: 10.1073/pnas.1222458110.

[23] Tapley, T.L., Franzmann, T.M., Chakraborty, S., Jakob, U. \& Bardwell, J.C. (2010). Protein refolding by pH-triggered chaperone binding and release. Proc Natl Acad Sci U S A. 107, 1071-1076. DOI: 10.1073/pnas.0911610107. 